\title{
Electrophysiological Biomarkers for Age-Related Changes in Human Atrial Cardiomyocytes: In Silico Study
}

\author{
Tatyana Nesterova ${ }^{1,2, *}$, Konstantin Ushenin ${ }^{1,2, * *}$, Dmitry Shmarko ${ }^{1,2,}$, and Olga \\ Solovyova ${ }^{1,2, * * *}$ \\ ${ }^{1}$ Institute of Immunology and Physiology of the Ural Branch of the RAS, Ekaterinburg, Russia \\ ${ }^{2}$ Ural Federal University, Ekaterinburg, Russia
}

\begin{abstract}
Age-related changes in human cardiomyocytes are closely related to cardiac diseases, especially atrial fibrillation. Restricted availability of biological preparations from the human atrial myocardium complicates experimental studies on the aging processes in cardiomyocytes. In this preliminary study, we used available experimental data on the age-related changes in ionic conductances in canine atrial cardiomyocytes to predict possible consequences of similar remodeling in humans using two mathematical models (Courtemanche98 and Maleckar09) of human atrial cardiomyocytes. The study was performed using the model population approach, allowing one to assess variability in the cellular response to different interventions affecting model parameters. Here, this approach was used to evaluate the effects of age-related parameter modulation on action potential biomarkers in the two models. Simulation results show a significant decrease in the action potential duration and membrane potential at $20 \%$ of the action potential duration in aging. These model predictions are consistent with experimental data from mammalians. The action potential characteristics are shown to serve as notable biomarkers of age-related electrophysiological remodeling in human atrial cardiomyocytes. A comparison of the two models shows different behavior in the prediction of repolarization abnormalities.
\end{abstract}

\section{Introduction}

Atrial fibrillation is one of the most common diseases in the human population and is widespread among seniors. The electrophysiological changes in human atrial cardiomyocytes in the aging heart may be strongly related to arrhythmia occurrence and sustainability. However, experimental observation of age-related changes in human atrial cardiomyocytes is complicated by ethical reasons and the restricted availability of healthy myocardial preparations from the human heart, especially of different ages.

In this preliminary study, we used available experimental data on age-related changes in ionic conductances in canine atrial cardiomyocytes $[1,2]$ to predict possible consequences

\footnotetext{
*This work was supported by RFBR \#18-015-00368, IIP UrB RAS theme \#AAAA-A18-118020590031-8, RF Government Act \#211 of March 16, 2013, the Program of the Presidium RAS.

**e-mail: konstantin.ushenin@urfu.ru

***e-mail: soloveva.olga@urfu.ru
} 
Table 1. Parameters of the normal action potential (AP) in human atrial cardiomyocytes. The last column shows parameters that were chosen for the classification of normal and pathological action potential in the current study. APD20, APD50, APD90 - action potential duration at X\% of repolarization, APA - action potential amplitude, RMP - resting membrane potential, V20 - voltage at $20 \%$ of action potential duration.

\begin{tabular}{llll}
\hline article & Sánchez et al. 2014 [6] & Muszkiewicz et al. 2018 [5] & Our populations \\
\hline APD20 (ms) & $1-60$ & $0.7-11.5$ & $0.7-60$ \\
APD50 (ms) & $6-200$ & $4.9-34.1$ & $4.9-200$ \\
APD90 (ms) & $190-440$ & $68.9-142.8$ & $68.9-440$ \\
APA (mV) & $75-120$ & $94.6-137.5$ & $75-137.5$ \\
RMP (mV) & $-85--65$ & $-85.2--66.1$ & $-85.2--65$ \\
V20 (mV) & $-35-10$ & - & $-35-10$ \\
\hline
\end{tabular}

of similar remodeling in humans using two mathematical models of human atrial cardiomyocytes: Courtemanche98 [3] and Maleckar09 [4]. The main goal of this analysis was to define the most important electrophysiological biomarkers for age-related changes in human atrial cardiomyocytes. The study was performed using the model population approach [5] to analyze the effects of age-related variation of model parameters on observed model behavior. We also compared the aging model populations based on the two ionic models of atrial cardiomyocytes.

\section{Methods}

We used Courtemanche98 [3] and Maleckar09 [4] ionic models for analysis of the electrophysiological consequences of cardiomyocyte aging. These models are widely used in computational electrophysiology studies.

There are essential differences between the models. Both models simulate human atrial action potential, but they produce the action potentials of different shapes. This is a result of different definitions of ionic currents in the models.

Both models are based on experimental data on the human atrial myocardium from patients of mostly the senior age group. Particularly, parameters of the ionic currents in the models were derived from experimental data recorded in the myocardium of patients of the following ages: $62 \pm 1$ years old in [7], 59 \pm 2 years old in [8], 61 to 78 years old in [9], 61 \pm 1 years old in [10], 63.9 \pm 10.7 years old in [11], $41-78$ years old in [12], and $45-71$ years old in [13]. Thus, we may consider the two mathematical models as reference models representing human atrial cardiomyocytes of the senior age group.

Almost no systematic data on the action potential in human cardiomyocytes from the adult or young adult group and no data on progressive changes in age-related cardiomyocyte characteristics throughout the aging process are available at the moment. On the contrary, such data is available for the canine atrium, as presented in Table 2. Based on this information, we chose several experimentally defined age-modified parameters of ionic currents to evaluate the effects of their variation on the action potential characteristics in human atrial models. We varied five parameters for the $\mathrm{Na}^{+}$current $\left(I_{N a}\right)$, transient outward $\mathrm{K}^{+}$current $\left(I_{t o}\right)$, and L-type $\mathrm{Ca}^{2+}$ current $\left(I_{C a L}\right)$ : three maximal conductances $g_{N a}, g_{t o}, g_{C a L}$, and two kinetic parameters of $I_{t o}$ - half-maximal voltage $V_{0.5}\left(I_{t o}\right)$, and the slow time constant of the recovery from inactivation $\tau_{\text {slowi }}\left(I_{t o}\right)$.

This study used the population of models methodology to investigate the behavior of the human atrial action potential with accounting for natural variability in cellular activity even within one testing group. An entire population of atrial action potential models was built with 
the variation of each of the five parameters within the range 0-200\% from the reference value $(100 \%)$ assigned in the original articles [3, 4]. Thus, each model from the population can be described by a five-dimensional scaling parameter vector, the values of which are expressed in percentage of the reference values. The population includes 10,000 models, each of which was pre-paced for 99 cycles at $1 \mathrm{~Hz}$ frequency to reach the steady-state.

The last action potential shape in each model was classified into one of the following groups: normal action potential (AP), pathological AP, early afterdepolarizations (EADs), delayed afterdepolarizations (DADs), as suggested in [14]. The models were experimentally calibrated according to the physiologically plausible ranges of AP biomarkers reported in the literature, as defined in Table 1. Color scales were used to display the phenotype of each tested model sample within the entire population on the planes of paired input parameter space (Figure 1).

To analyze age-related changes in human atrial model behavior, we proposed five aging sub-populations of models selected from the entire model population: Adult I, Adult II,

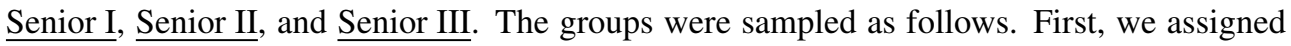
the reference parameters of human atrial models from $[3,4]$ as the mean values for the

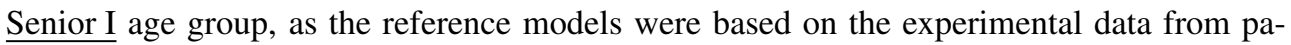
tients of around 60 years old. To define mean parameters for younger and older model sub-populations, we varied the five age-related parameters based on the experimental data on the relative changes in the parameters between the Senior I and the Adult I groups and our assumption on the linear change in the parameters in aging. The direction and percentage of age-related modulation in the mean parameter values between the Adult I and Senior I groups was set according to the experimental data from the canine atrial myocardium from 1-3 (dult 1) and 8 (Senior I) years old dogs (see Table 2). Then we connected the mean points in the 5-dimensional parameter space by the "aging line" used to compute the mean parameters for the intermediate Adult II group between the Adult I and Senior I groups and for the older groups Senior II and Senior III that lay in the opposite direction from the Senior I group on the aging line (see Table 2 for the mean parameter values in the groups expressed in percentage of the reference values in the Senior I, and the lines in the parameter spaces shown in Figure 1).

The age sub-populations of models were sampled from the sphere in the parameter space around the mean parameter vector for the corresponding group at a distance less than $30 \%$ of the length of the reference 5-variable parameter vector (see sub-population circles on the 2-parameter planes shown in Fig. reffig:projections). Each sub-population consisted of 1,080 to 2,070 models.

Age-related biomarkers for AP shape distinguishing between the groups were then derived using a naive Bayesian classifier.

\section{Results}

Figure 1 shows the projections of the five-dimensional space of varied age-related parameters to two-dimensional planes. The color of each point indicates the AP's class according to the used model classification. As can be seen from Figure 1, the two atrial cardiomyocyte models we tested are differently vulnerable for the repolarization abnormalities within the tested parameter range. The much higher percentage of EAD was generated by the Maleckar09 model, and no DAD was produced by the Courtemanche98 model. The distribution of the models between the different classes within the parameter space is also essentially different between the cellular reference models. Particularly for the Maleckar09 model, EADs and DADs are produced in a narrow range of $g_{C a L}$ of high average value, but with a more wider distribution of the parameters of $I_{t o}$ compared to the Courtemanche98 model. 
Table 2. Experimental data on age-related changes in the transmembrane currents in canine atrial myocardium.

\begin{tabular}{llllll}
\hline current & parameter & change with age & article & age (year) & atrium \\
\hline$I_{\text {Ca,L }}$ & & $\downarrow 47 \%$ & {$[1]$} & $2-5 \&>8$ & RA \\
& \multirow{2}{*}{ density } & $\downarrow 43 \%$ & {$[2]$} & $2-2.5 \&>8$ & LA \\
& & $\downarrow 43 \%$ & {$[15]$} & $1-3 \&>8$ & LA \\
& & $\downarrow 37 \%$ & & & \\
\hline \multirow{4}{*}{$I_{t o}$} & density & $\uparrow 48 \%$ & & & \\
& $V(0.5)_{i}$ & $\downarrow 15 \%$ & & & \\
& $\tau_{\text {fast }_{i}}$ & $\uparrow 68 \%$ & & & \\
& $\tau_{\text {slow }_{i}}$ & $\uparrow 246 \%$ & & & \\
\hline \multirow{2}{*}{$I_{N a}$} & density & $\uparrow 28 \%$ & \multirow{2}{*}[16]{} & \multirow{2}{*}{$2-5 \&>8$} & RA \\
& & $\uparrow 22 \%$ & & & LA \\
\hline
\end{tabular}

Table 3. The linear model of age-related changes in human atrial cardiomyocytes. The numbers show parameter values in the aging groups expressed in percentage of the reference values assigned for the

Senior I age group. Parameter values in other groups are based on the experimental data and assumption on the liner change in the parameters between the aging groups.

\begin{tabular}{|c|c|c|c|c|c|c|c|}
\hline & & $\begin{array}{l}\text { Courtemanche } 98 \\
\text { Maleckar09 }\end{array}$ & $\underline{\text { Adult I }}$ & $\underline{\text { Adult II }}$ & $\underline{\text { Senior I }}$ & $\underline{\text { Senior II }}$ & $\underline{\text { Senior III }}$ \\
\hline $\begin{array}{l}I_{C a, L} \\
{[1]}\end{array}$ & $g_{C a, L}$ & $\begin{array}{l}0.12375 \mathrm{mS} / \mathrm{uF} \\
0.135 \mathrm{mS} / \mathrm{uF}\end{array}$ & $189 \%$ & $144.5 \%$ & $100 \%$ & $55.5 \%$ & $11 \%$ \\
\hline \multirow{3}{*}{$\begin{array}{l}I_{t o} \\
{[1]}\end{array}$} & $g_{t o}$ & $\begin{array}{l}0.1652 \mathrm{mS} / \mathrm{uF} \\
0.150 \mathrm{mS} / \mathrm{uF}\end{array}$ & $68 \%$ & $84 \%$ & $100 \%$ & $116 \%$ & $132 \%$ \\
\hline & $\tau_{i}$ & & $29 \%$ & $64.5 \%$ & $100 \%$ & $135.5 \%$ & $171 \%$ \\
\hline & $V(0.5)_{i}$ & $\begin{array}{l}-43.1 \mathrm{mV} \\
-40.5 \mathrm{mV}\end{array}$ & $118 \%$ & $109 \%$ & $100 \%$ & $91 \%$ & $82 \%$ \\
\hline $\begin{array}{l}I_{N a} \\
{[16]}\end{array}$ & $\begin{array}{l}g_{N a} \\
P_{N a}\end{array}$ & $\begin{array}{l}7.8 \mathrm{mS} / \mathrm{uF} \\
0.0018 \mathrm{nl} / \mathrm{s}\end{array}$ & $78 \%$ & $89 \%$ & $100 \%$ & $111.5 \%$ & $123 \%$ \\
\hline
\end{tabular}

The series of AP shapes without repolarization abnormalities generated in the agedependent sub-populations are shown in Figure 2. The linear model of the age-related parameter modulation that we used predicts qualitatively similar changes in the AP characteristics for both Courtemanche 98 and Maleckar09 models. The most notable age-related changes are observed for the action potential duration at $90 \%$ of repolarization (APD90) and action potential voltage at $20 \%$ of APD (V20) (Figure 2), which both decrease with aging. In the Courtemanche98 model, APD90 does not significantly change between Adult I, II and Senior I age groups and linearly decreases between the Senior I and Senior III groups. In the Maleckar09 model, there is a gradual logistic decrease in the APD90. The V20 dependence on age is similar between the models.

There are several differences in the age-related effects on other AP biomarkers between the models. The Maleckar09 model predicts decreasing resting potential during aging, but the Courtemanche 98 model does not. Note also, the Courtemanche 98 model produces AP with the spike-and-dome morphology in every aging group of models except the Senior III. Conversely, the Maleckar09 models display this AP morphology in only the Senior III age groups of models. Both models show almost no effect of age-related parameter modulation on 

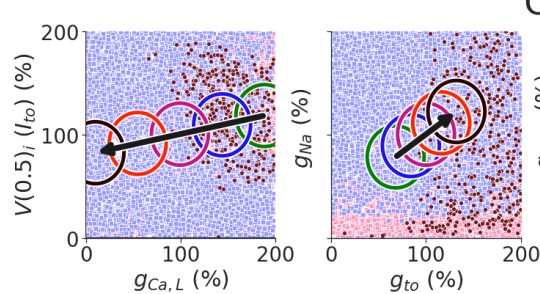

Courtemanche 98

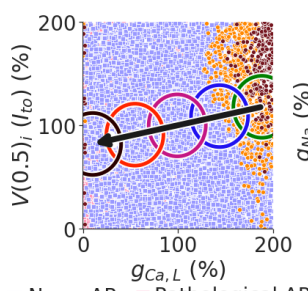

Norm AP Pathological AP
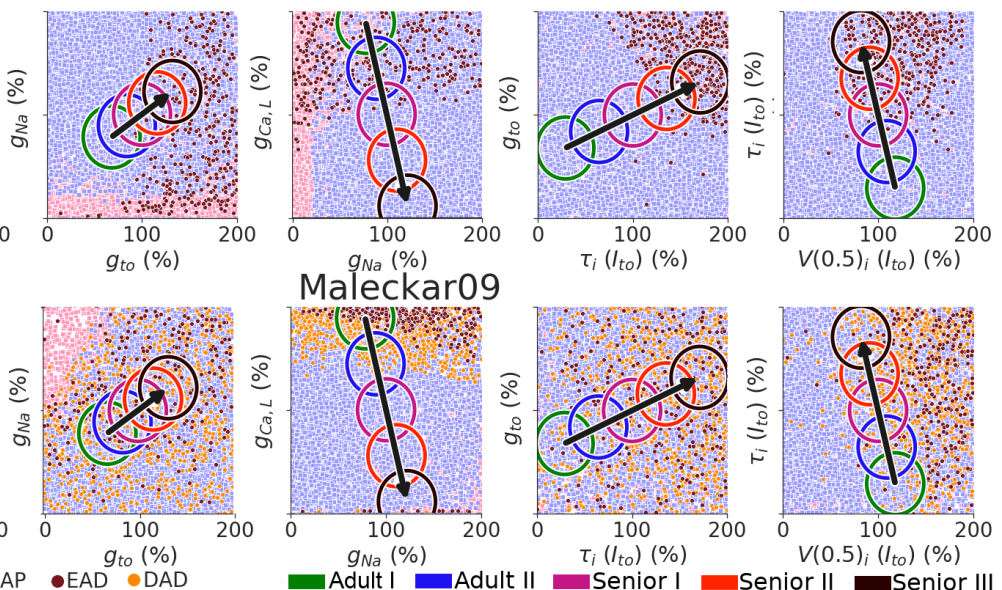

Figure 1. Projection of the five-dimensional parameter space on the two-dimensional planes. Colour points show the classification of APs for the model samples. Age-dependent model sub-populations are shown as the circles on the 2-parameter planes. The black arrow shows the direction of the age-related changes in the models of human atrial cardiomyocytes.
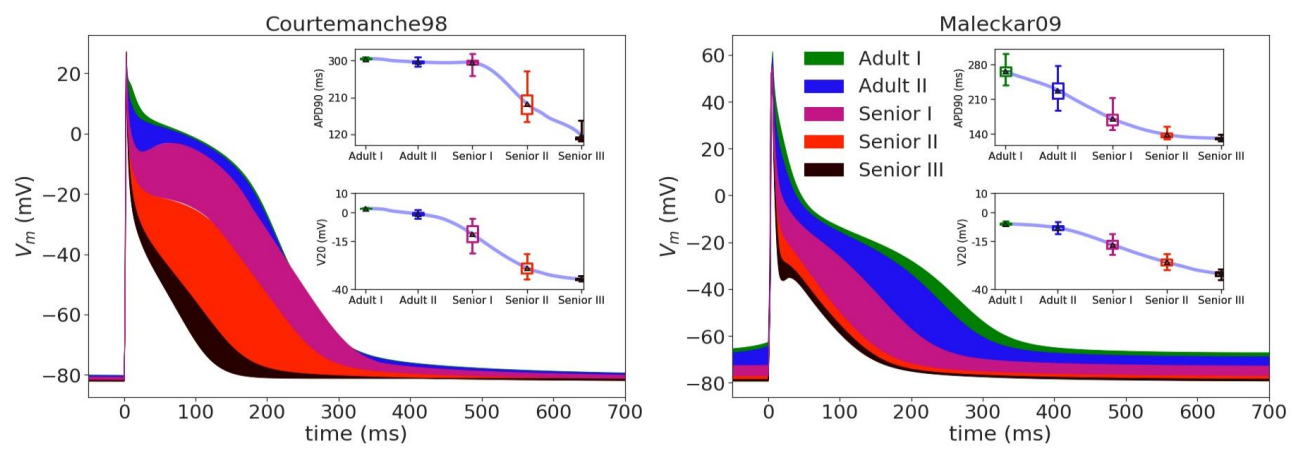

Figure 2. Age-related changes in the action potential of cardiomyocytes. Each color shows one of the age sub-populations. Insert plots show the distribution of APD90 and V20 in the sub-populations.

the absolute value of the AP upstroke and height. Adult II models differ from Senior I models for the V20 parameter: $-7.93 \pm 1.26$ versus $-16.58 \pm 2.73 \mathrm{mV}$ in the Maleckar09 model, and $0.72 \pm 0.99$ versus $-11.23 \pm 4.98 \mathrm{mV}$ in the Courtemanche98 model. Senior I models differ from Senior II models for the APD90 parameter: $170.97 \pm 13.97$ versus $138.77 \pm 5.51 \mathrm{~ms}$ in the Maleckar09 model, and 295.12 \pm 7.74 versus $195.13 \pm 29.94 \mathrm{~ms}$ in the Courtemanche98 model. Other parameters of the AP show insignificant differences between the ages in the absolute physical units.

Our goal was to provide most reliable biomarkers of cardiomyocites aging that are based on AP contours. The naive Bayes classifier was able to distinguish model behaviour between the groups and provided the most significant border values related to the age-groups. The

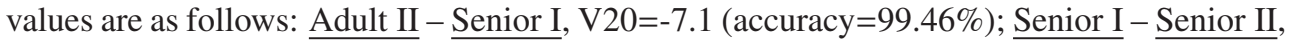




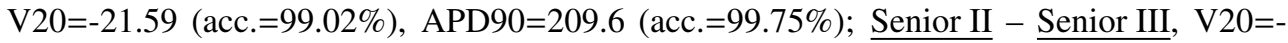
31.7 (acc. $=85.24 \%)$. Other linear rules show accuracy lower than $85 \%$.

\section{Discussion}

Experimental studies on isolated canine atrial cardiomyocytes show prolongation of the APD90 in aging ([1, 2]). Several studies on the whole human atriums reported an increase in the effective refractory period associated with the age $[17,18]$. These observations also suggest an increase in APD90. This data disagrees with our predictions on the APD90 shortening in aging models of human atrial cardiomyocytes suggesting the inter-species difference in the aging processes in atrial cardiomyocytes.

The decrease in V20 in the aging models is consistent with experimental observations in canine atrial cardiomyocytes ([1,2]). This AP biomarker is shown to closely correlate with parameters of the L-type calcium current and transient outward current, which were directly modified in the aging sub-populations, while APD90 is sensitive to the variation in all transmembrane currents under consideration.

In summary, we suppose that V20 and APD90 are the most trustworthy electrophysiological biomarkers of human atrial cardiomyocyte aging. Both of them show significant changes during in aging in the absolute values of physical units. The change in V20 is consistent with experimental observations. However, APD90 is more suitable for experimental and clinical measurements, because it may be estimated from the activation-recovery interval of the unipolar electrogram.

The presented study shows preliminary results using the model population approach that may be further extended in several directions. Currently, we are working on a more specific definition of age-related model groups. The proposed simple linear approach we used here to simulate age-related changes in model parameters has to be verified in tissue models against available clinical information on age-related changes in intracardiac electrograms and electrocardiograms. Also, we see potential applications of our approach for drug-testing with accounting for age-related changes in cellular activity.

\section{Conclusion}

In this preliminary study, we used experimental data on the age-related changes in canine atrium cardiomyocytes to build aging populations of models based on the two models of human atrial cardiomyocyte (Courtemanche98 and Maleckar09). The populations of models identify APD90 and V20 as significant biomarkers related to age-dependent changes in human atrial cardiomyocytes. We suppose that distinguishing aging groups in the human population may be classified according to the found biomarkers.

\section{References}

[1] W. Dun, T. Yagi, M.R. Rosen, P.A. Boyden, Cardiovascular Research 58, 526 (2003)

[2] T.Y. Gan, W. Qiao, G.J. Xu, X.H. Zhou, B.P. Tang, J.G. Song, Y.D. Li, J. Zhang, F.P. Li, T. Mao et al., Experimental and therapeutic medicine 6, 919 (2013)

[3] M. Courtemanche, R.J. Ramirez, S. Nattel, American Journal of Physiology-Heart and Circulatory Physiology 275, H301 (1998)

[4] M.M. Maleckar, J.L. Greenstein, W.R. Giles, N.A. Trayanova, American Journal of Physiology-Heart and Circulatory Physiology 297, H1398 (2009) 
[5] A. Muszkiewicz, X. Liu, A. Bueno-Orovio, B.A. Lawson, K. Burrage, B. Casadei, B. Rodriguez, American Journal of Physiology-Heart and Circulatory Physiology 314, H895 (2018)

[6] C. Sánchez, A. Bueno-Orovio, E. Wettwer, S. Loose, J. Simon, U. Ravens, E. Pueyo, B. Rodriguez, PloS one 9, e105897 (2014)

[7] J. Feng, G. Li, B. Fermini, S. Nattel, American Journal of Physiology-Heart and Circulatory Physiology 270, H1676 (1996)

[8] A.J. Workman, K.A. Kane, A.C. Rankin, Cardiovascular research 52, 226 (2001)

[9] P. Gluais, M. Bastide, D. Grandmougin, G. Fayad, M. Adamantidis, European journal of pharmacology 497, 215 (2004)

[10] Z. Wang, J. Feng, H. Shi, A. Pond, J.M. Nerbonne, S. Nattel, Circulation Research 84, 551 (1999)

[11] L. Firek, W. Giles, Cardiovascular research 30, 31 (1995)

[12] Z. Wang, B. Fermini, S. Nattel, Cardiovascular research 28, 1540 (1994)

[13] A.A. Dawodu, F. Monti, K. Iwashiro, M. Schiariti, R. Chiavarelli, P.E. Puddu, International journal of cardiology 54, 237 (1996)

[14] A. Muszkiewicz, O.J. Britton, P. Gemmell, E. Passini, C. Sánchez, X. Zhou, A. Carusi, T.A. Quinn, K. Burrage, A. Bueno-Orovio et al., Progress in biophysics and molecular biology 120, 115 (2016)

[15] G.J. Xu, T.Y. Gan, B.P. Tang, Z.H. Chen, A. Mahemuti, T. Jiang, J.G. Song, X. Guo, Y.D. Li, X.H. Zhou et al., Molecular medicine reports 8, 1343 (2013)

[16] S. Baba, W. Dun, M. Hirose, P.A. Boyden, American Journal of Physiology-Heart and Circulatory Physiology 291, H756 (2006)

[17] P.M. Kistler, P. Sanders, S.P. Fynn, I.H. Stevenson, S.J. Spence, J.K. Vohra, P.B. Sparks, J.M. Kalman, Journal of the American College of Cardiology 44, 109 (2004)

[18] Y.K. Lin, Y.A. Chen, T.I. Lee, Y.C. Chen, S.A. Chen, Y.J. Chen, Circulation Journal 82, 1237 (2018) 